\title{
Coil migration into the common bile duct after embolization of a hepatic artery pseudoaneurysm
}

A 65-year-old woman was referred to our hospital for thoracic spine metastasis. She had undergone embolization 8 years ago for hemobilia caused by a pseudoaneurysm in the right hepatic artery, which had developed after cholecystectomy with T-tube choledochostomy. Laboratory studies revealed alanine aminotransferase $510 \mathrm{IU} / \mathrm{L}$, total/direct bilirubin 3.69/ $3.22 \mathrm{mg} / \mathrm{dL}$, alkaline phosphatase $496 \mathrm{U} / \mathrm{L}$, and $\gamma$-glutamyltransferase $704 \mathrm{U} / \mathrm{L}$. Abdominal computed tomography showed a high-density coil at the hepatic hilum, compatible with pseudoaneurysm after coil embolization, and marked dilatation of the bilateral intrahepatic bile ducts (๑ Fig. 1).

Percutaneous transhepatic biliary drainage (PTBD) was carried out because of obstructive jaundice. Endoscopic retrograde cholangiopancreatography revealed dilatation of the intra- and extrahepatic bile ducts with amorphous filling defects in the upper part of the common bile duct (CBD), and five microcoils around the hepatic hilum ( $\bullet$ Fig. 2 ).

Endoscopic papillary balloon dilation (EPBD) was carried out, and a Dormia basket was inserted and several mixed CBD stones extracted ( $\bullet$ Fig. 3 ).

A microcoil was found inside a fragment of a removed stone ( Fig. 4).

Following this, the PTBD was removed and the patient discharged in a relatively stable condition.

Hemobilia most often is due to iatrogenic causes and accidental trauma, followed by gallstones, inflammation, vascular malformations, and tumors [1]. The common symptoms are upper gastrointestinal hemorrhage, upper abdominal pain, and jaundice. To our knowledge, there has been only one prior report of hemobilia caused by hepatic artery pseudoaneurysm after T-tube choledochostomy [2]. Mechanical compression of the bile duct mucosa and the adjacent part of the hepatic artery by the T-tube may lead to blood vessel erosion and formation of a pseudo-

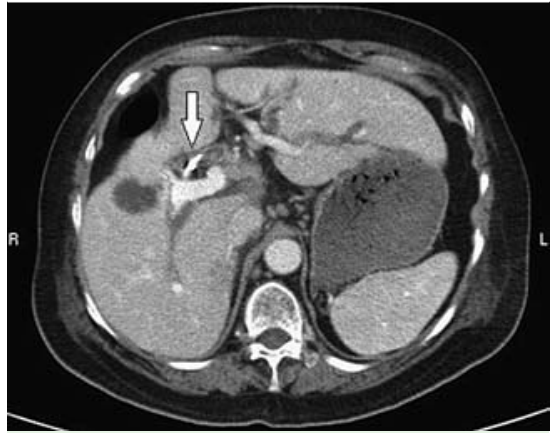

Fig. 1 High-density coil at the hepatic hilum (arrow), compatible with a pseudoaneurysm after coil embolization and marked dilatation of the intrahepatic bile duct in an older woman with history of hemobilia.

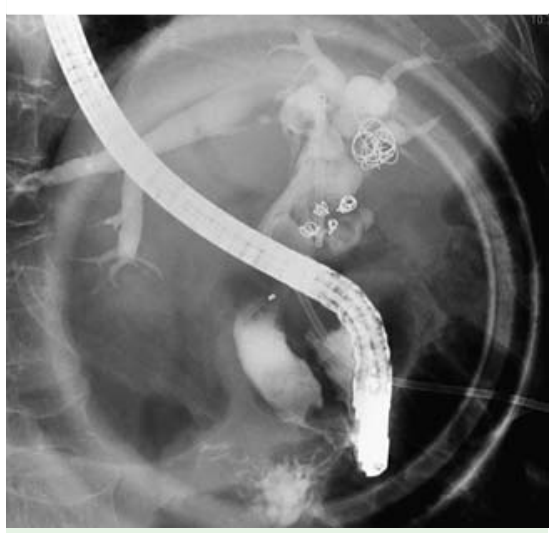

Fig. 2 Dilated intra- and extrahepatic bile ducts with amorphous filling defects visualized in the upper part of the common bile duct. Five microcoils around the hepatic hilum and the percutaneous transhepatic biliary drainage catheter are also seen.

aneurysm. Migration of coils into the bile duct through a small arterial-biliary fistula after embolization of a hepatic artery pseudoaneurysm is a rare complication [3], and may trigger or exacerbate the formation of CBD stones, with cholangitis.

Endoscopy_UCTN_Code_CCL_1AZ_2AI

Competing interests: None
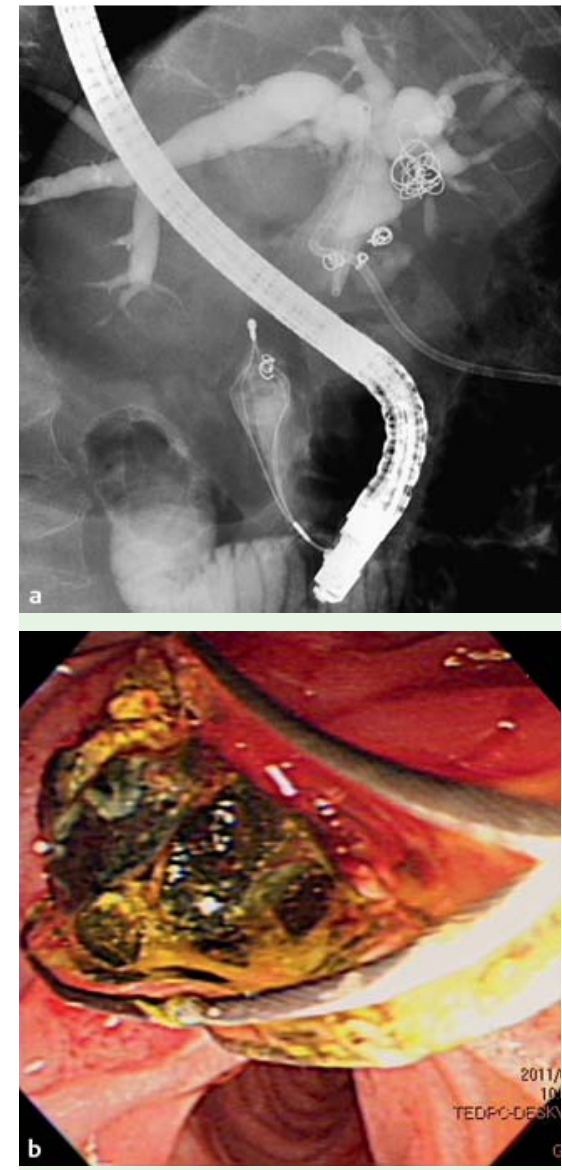

Fig. 3 Endoscopic papillary balloon dilation followed by insertion of a Dormia basket to remove a microcoil (a) and several common bile duct stones (b).

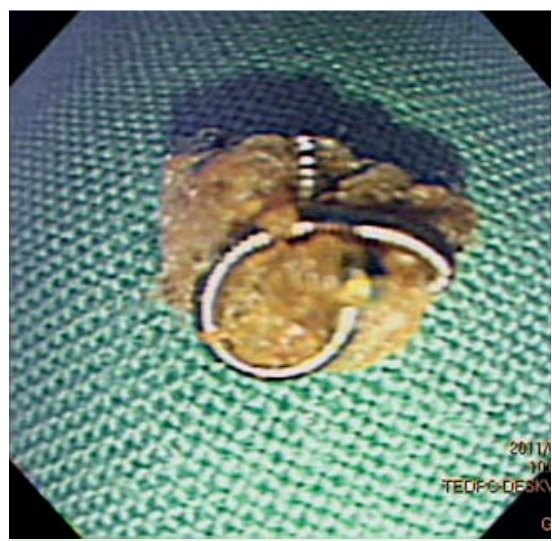

Fig. 4 A microcoil within a fragment of a removed stone. 
W. Y. Kao' ${ }^{1}$, Y. Y. Chiou, ${ }^{2,3}$, T. S. Chen ${ }^{1,3}$ 1 Division of Gastroenterology, Department of Medicine, Taipei Veterans General Hospital, Taipei, Taiwan

2 Division of Gastrointestinal Radiology, Department of Radiology, Taipei Veterans General Hospital, Taipei, Taiwan

3 Faculty of Medicine, School of Medicine, National Yang-Ming University, Taipei, Taiwan

\section{References}

1 Green MH, Duell RM, Johnson CD, Jamieson NV. Haemobilia. Br J Surg 2001; 88: $773-$ 786

2 Lee YT, Lin H, Chen KY et al. Life-threatening hemobilia caused by hepatic pseudoaneurysm after T-tube choledochostomy: report of a case. BMC Gastroenterol 2010; 10: 81

3 Ozkan OS, Walser EM, Akinci D et al. Guglielmi detachable coil erosion into the common bile duct after embolization of iatrogenic hepatic artery pseudoaneurysm. J Vasc Interv Radiol 2002; 13: 935-938
Bibliography

DOI $10.1055 / \mathrm{s}-0030-1256687$

Endoscopy 2011; 43: E364-E365

(c) Georg Thieme Verlag KG Stuttgart · New York . ISSN 0013-726X

\section{Corresponding author}

\section{T. S. Chen}

Division of Gastroenterology

Department of Medicine

Taipei Veterans General Hospital

Taipei

Taiwan

Fax: +886-2-2873-9318

tschen@vghtpe.gov.tw 